\title{
Respiratory-Induced Hemodynamic Changes Measured by Whole-Body Multichannel Impedance Plethysmography
}

\section{P. LANGER ${ }^{1,2}$, P. JURÁK ${ }^{1}$, V. VONDRA ${ }^{1,2}$, J. HALÁMEK $^{1}$, M. MEŠŤANÍK ${ }^{3,4}$ I. TONHAJZEROVÁ ${ }^{4,3}$, I. VIŠČOR ${ }^{1}$, L. SOUKUP ${ }^{2}$, M. MATEJKOVA ${ }^{2}$, E. ZÁVODNÁ ${ }^{5,6}$, P. LEINVEBER ${ }^{2}$}

${ }^{1}$ Institute of Scientific Instruments of the Czech Academy of Sciences, Brno, Czech Republic, ${ }^{2}$ International Clinical Research Center, St. Anne's University Hospital, Brno, Czech Republic, ${ }^{3}$ Biomedical Center Martin, Jessenius Faculty of Medicine in Martin, Comenius University in Bratislava, Martin, Slovak Republic, ${ }^{4}$ Department of Physiology, Jessenius Faculty of Medicine in Martin, Comenius University in Bratislava, Martin, Slovak Republic, ${ }^{5}$ Department of Physiology and Pathophysiology, Faculty of Medicine, University of Ostrava, Ostrava, Czech Republic, ${ }^{6}$ Department of Psychology, Faculty of Arts, Masaryk University, Brno, Czech Republic

Received October 16, 2017

Accepted January 19, 2018

On-line May 10, 2018

\section{Summary}

The cardiovascular system is described by parameters including blood flow, blood distribution, blood pressure, heart rate and pulse wave velocity. Dynamic changes and mutual interactions of these parameters are important for understanding the physiological mechanisms in the cardiovascular system. The main objective of this study is to introduce a new technique based on parallel continuous bioimpedance measurements on different parts of the body along with continuous blood pressure, ECG and heart sound measurement during deep and spontaneous breathing to describe interactions of cardiovascular parameters. Our analysis of 30 healthy young adults shows surprisingly strong deep-breathing linkage of blood distribution in the legs, arms, neck and thorax. We also show that pulse wave velocity is affected by deep breathing differently in the abdominal aorta and extremities. Spontaneous breathing does not induce significant changes in cardiovascular parameters.

\section{Key words}

Bioimpedance • Blood flow • Blood volume • Pulse wave velocity - Respiration

\section{Corresponding author}

P. Langer, Institute of Scientific Instruments of the Czech Academy of Sciences, Královopolská 147, Brno, Czech Republic. E-mail: peter.langer@seznam.cz

\section{Introduction}

The cardiovascular system is characterized by continuous dynamic changes of the activity of the heart and vessels according to the demands of organ systems and outputs from the complex central neuroendocrine regulation. These functional changes can be described by common hemodynamic parameters such as heart rate (HR), stroke volume (SV), cardiac output (CO) and blood pressure (BP), and additional information can also be obtained from detailed analysis of regional blood flow. However, application of conventional methods for the assessment of blood flow and distribution characteristics simultaneously in different body parts is largely limited by invasiveness (thermodilution methods), low accuracy (e.g. photoplethysmography), the cost of the examination (e.g. phase-contrast magnetic resonance imaging, positron emission tomography) or methodological difficulties with simultaneous multisite recording (e.g. Doppler ultrasound methods) (McRobbie et al. 2007, Leenders 1994, Allen 2007, Bagger 1984, Azran et al. 2004).

Recently, a noninvasive bioimpedance method based on changes of the electrical impedance of a body segment measured using high-frequency (HF) current and 
surface electrodes $(20-150 \mathrm{kHz})$ has attracted attention as a promising approach in the study of hemodynamics (Bernstein 2010). In general, blood is better HF current conductor when compared to other tissues, e.g. muscles, bones and fat, and the bioimpedance decreases with greater blood volume constituting a mechanism known as the volume effect. Moreover, the impedance of blood also fluctuates in accordance with blood flow. The red blood cells orient themselves in the direction of flow and form less conductive paths in the highly conductive blood plasma, and the bioimpedance decreases. When the flow stops, the red blood cells orient themselves randomly again, the less conductive paths disappear, and the bioimpedance increases - what is known as the orientation effect (Bernstein 2010). The relative contribution of both effects is comparable (Visser et al. 1990, Visser et al. 1993). In relation to cardiac cycle and postural changes, oscillations of blood volume and flow velocity vary with different patterns across the body. With respect to time-dependent dynamics, slow changes of bioimpedance ( $\mathrm{Z} 0)$ are considered to reflect the amount of blood in the measured body part, and the negative maximum of fast changes of bioimpedance within the cardiac cycle $\left(-d Z j / d t_{\max }\right)$ is considered an electrical analogue of the maximum volume change interpreted as flow parameter (Bernstein 2010). The time interval between $-d Z j / d t_{\max }$ positions at two body locations is used to calculate the pulse wave velocity (PWV); a higher PWV corresponds to higher arterial stiffness (Sutton-Tyrrell et al. 2005).

Importantly, most of the studies based on conventional methods for the assessment of blood-flow properties predominantly evaluate regional characteristics within a selected part of the circulation or only specific parameters without simultaneous assessment of complex cardiovascular functioning. This limits the detailed understanding of physiological mechanisms behind the dynamic changes of blood distribution in the whole body. Therefore, we aimed to introduce a new technique for simultaneous assessment of blood flow and distribution characteristics in different parts of the body based on parallel continuous multisite measurement of the bioimpedance signal together with noninvasive continuous BP assessment, 12-lead electrocardiogram (ECG) and heart sounds recording in response to resting and paced deep breathing.

\section{Materials and Methods}

\section{Subjects}

The studied population consisted of 30 healthy non-smoking volunteers (15 men/15 women) aged 20-36 years. The characteristics of the group are summarized in Table 1. The study was approved by the St. Anne's University Hospital Ethics Committee, and all the participants gave informed written consent to their involvement in the study prior to the examination.

Table 1. Subjects' characteristics.

$\begin{array}{lc}\text { Age (years) } & 23.1 \pm 4.5 \\ \text { Males/Females }(\mathrm{n}) & 15 / 15 \\ \text { Height }(\mathrm{cm}) & 179 \pm 6 \\ \text { Weight }(\mathrm{kg}) & 73 \pm 12 \\ \text { SBP }(\mathrm{mm} \mathrm{Hg}) & 136 \pm 34 \\ \operatorname{DBP}(\mathrm{mm} \mathrm{Hg}) & 73 \pm 22 \\ M B P(\mathrm{~mm} \mathrm{Hg}) & 94 \pm 26 \\ R R(\mathrm{~s}) & 0.94 \pm 0.12 \\ B M I\left(\mathrm{~kg} / \mathrm{m}^{2}\right) & 22.0 \pm 2.7\end{array}$

SBP - systolic blood pressure, DBP - diastolic blood pressure, MBP - mean blood pressure, RR - mean duration of RR-interval, BMI - body mass index. Values are expressed as mean \pm SD.

\section{Protocol}

All the measurements were performed under standard conditions in an air-conditioned soundattenuated laboratory (room temperature $22^{\circ} \mathrm{C}$, minimization of external stimuli) between $2 \mathrm{pm}$ and $5 \mathrm{pm}$. The subjects were asked to refrain from heavy exercise and administration of medicaments, supplements and stimulants potentially affecting cardiovascular or autonomic nervous system activity (including caffeinated or alcoholic beverages) at least $24 \mathrm{~h}$ before the test, and not to eat for at least $2 \mathrm{~h}$ before the examination. After application of surface impedance electrodes, ECG electrodes, a chest microphone for heart sounds recording and a finger cuff for continuous BP recording (position of each sensor illustrated in Figure 1A), the participants rested for $15 \mathrm{~min}$ in a supine position. Following this, the examination was performed in the supine position during 3 periods, each lasting $5 \mathrm{~min}$ : 1) spontaneous breathing; 2) deep ribcage breathing with a frequency of $0.1 \mathrm{~Hz}$ according to a breath metronome, duration of inhalation and exhalation phase $5 \mathrm{~s}$ each; 3 ) spontaneous breathing. 


\section{Data collection and pre-processing}

The multichannel bioimpedance signal was assessed using a whole-body Multichannel Bioimpedance Monitor (MBM) (Vondra et al. 2015) simultaneously with 12-lead ECG signal (ECG12, ISI Brno, Czech Republic), continuous photoplethysmographic arterial BP recording (Finapres-2300, Ohmeda Medical, Englewood, Co., USA), and heart sounds phonocardiographic (PCG) recording (PCG 1.0, ISI Brno, Czech Republic). All the signals were recorded with a sampling frequency of $500 \mathrm{~Hz}$ and a resolution of 16 bits. An antialiasing filter was used before sampling.

The MBM used three independent current sources ( $I_{1}, I_{2}$ and $I_{3}, \quad$ Fig. $1 A$ ), and measured bioimpedance simultaneously at 18 locations (arms, legs, neck and torso, Fig. 1A). To avoid interference from current sources, three different frequencies were used and the scanned channels were tuned to the selected source. The frequency bandwidth was $250 \mathrm{~Hz}$ for each channel and the current source frequencies were $49 \mathrm{kHz}, 50 \mathrm{kHz}$ and $51 \mathrm{kHz}$, with current root mean square $=1 \mathrm{~mA}$.

The MBM used a four-electrode method to measure bioimpedance for the desired part of the body. A current source $I i(t)$ with an alternating sine wave current was connected to the outer electrodes and the voltage-sensing channel $\mathrm{CHj}$ was connected to the inner electrodes. The bioimpedance $Z j(t)$ between electrodes $\mathrm{CH} \mathrm{j}+$ and $\mathrm{CH} j$ - was calculated based on Ohm's Law:

$$
Z 0 j(t)=\mathrm{Uj}(t) / I i(t)
$$

where $\mathrm{Uj}(t)$ is the voltage between electrodes $\mathrm{CH} \mathrm{j}+$ and $\mathrm{CH} \mathrm{j}$-. Bioimpedance is a complex value and can be separated into a real $\mathrm{Rj}(t)$ and imaginary $\mathrm{iXj}(t)$ part of bioimpedance:

$$
Z 0 j(t)=\mathrm{Rj}(t)+\mathrm{iXj}(t)
$$

This principle was applied to all 18 evaluated locations on the body (Fig. 1A). The following 12 locations were evaluated in this study: left and right carotid on the neck ( $\mathrm{Z} 1$ and $\mathrm{Z} 2)$, left and right part of the chest ( $\mathrm{Z} 3$ and $\mathrm{Z4}$ ), left and right thigh ( $\mathrm{Z} 5$ and $\mathrm{Z6})$, calves (Z7 and Z8), upper arms (Z13 and Z14) and forearms (Z15 and Z16).
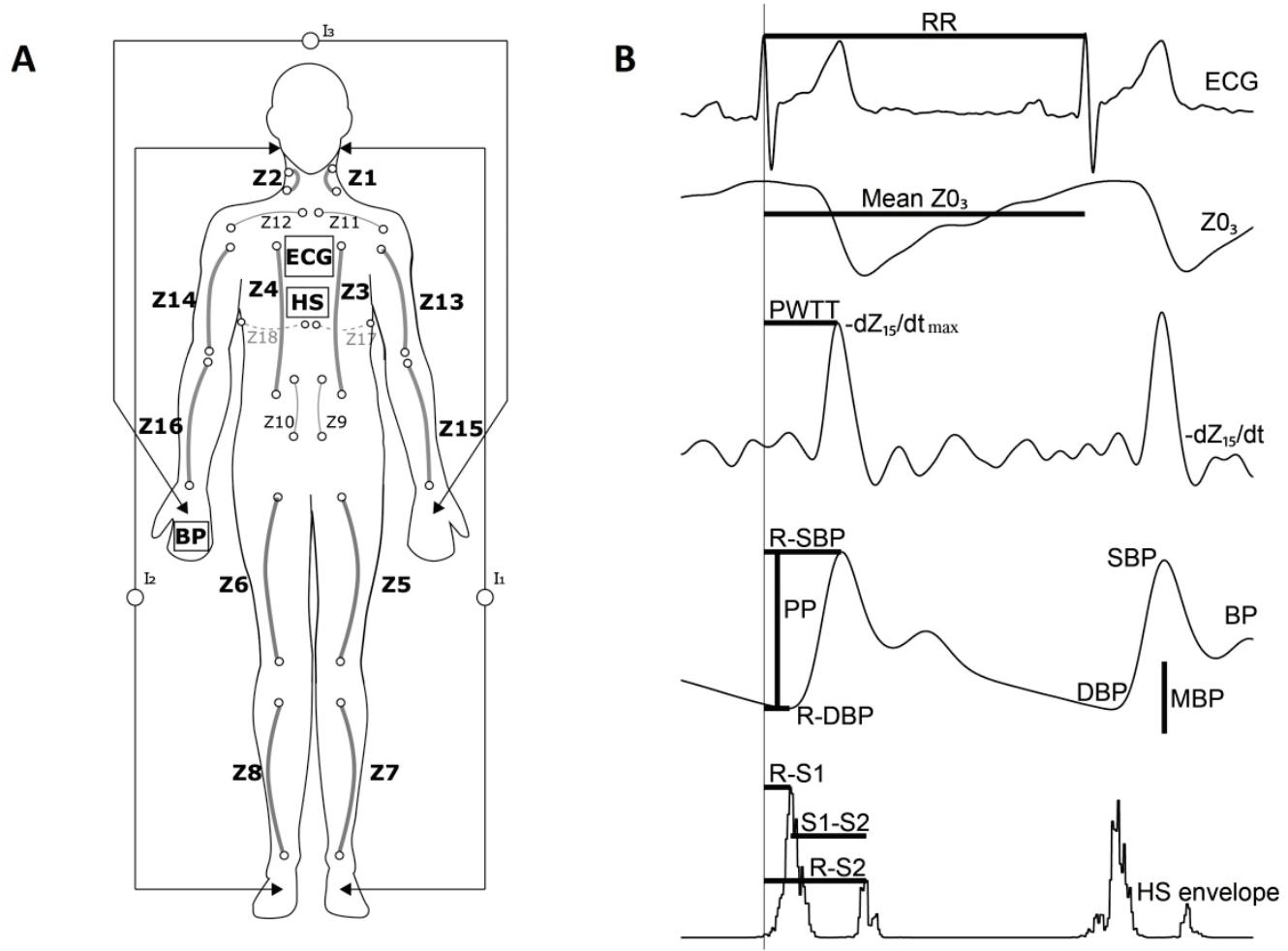

Fig. 1. A. Measurement locations of Multichannel Bioimpedance Monitor (MBM). Measurement locations are marked as $\mathrm{Zj}$ and current sources are marked as $\mathrm{I}_{\mathrm{i}}$. Locations of blood pressure (BP), ECG, and heart sounds (HS) sensors are also depicted in the figure. Bioimpedance channels evaluated in this study are marked in bold fond $(Z 1, Z 2, \ldots)$ and thick lines. B. Detection of hemodynamic parameters from ECG, impedance $Z 0 j$, derivate impedance $-\mathrm{dZj} / \mathrm{dt}$, blood pressure (BP) and heart sounds envelope curves. RR duration of RR interval, Mean $Z 0 \mathrm{j}$ - mean value of $\mathrm{ZO} \mathrm{j}$ within RR interval, $-\mathrm{dZj} / \mathrm{dt}_{\max }$ - maximum of negative derived impedance curve, PWTT - pulse-wave transit time, SBP - systolic BP, DBP - diastolic BP, PP - pulse pressure, the difference between DBP and SBP in one cardiac cycle, MBP - mean blood pressure, R-SBP - delay between R-wave and SBP, R-DBP - delay between R-wave and DBP, R-S1 delay between R-wave and S1, R-S2 - delay between R-wave and S2, S1-S2 - parameter similar to left ventricle ejection time. 
Phonocardiographic recordings were filtered individually for each person using wavelet transform filter bank db16. The signal was decomposed into 5 detail levels. Reconstruction was performed from all the combinations of consecutive details as described elsewhere (Langer et al. 2014). Every combination represents one frequency constrain of the heart sound one filter. Next, the subject's best filter was selected based on the highest correlation between the detected first heart sound distance from the R-wave and respiration. The highest correlation means the best detection of $\mathrm{S} 1$ (Langer et al. 2014). The power envelope was then computed. The S1 was detected as the center of gravity of the envelope between the R-wave and 0.2 length of the cardiac cycle. The S2 was detected as the center of gravity of the envelope between 0.3 length of the cardiac cycle and 0.7 length of the cardiac cycle.

Computation of bioimpedance hemodynamic and cardiovascular parameters

To describe the respiratory-induced dynamic activity of the circulatory system, the following parameters were evaluated for every cardiac cycle from selected bioimpedance channels (j), blood pressure, heart sounds and ECG recordings.

Mean $\mathrm{Z0j}$ : mean value of $\mathrm{Z} 0 \mathrm{j}$ within cardiac cycle. The bioimpedance signal was filtered by a low-pass filter with a cut-off frequency of $0.75 \mathrm{~Hz}$ to eliminate heartbeat influence. While $80 \%$ of blood mass is concentrated in the veins, $\mathrm{Z} 0 \mathrm{j}$ reflects slow changes of the amount of blood in the measured location, where the lower the $\mathrm{Z} 0 \mathrm{j}$ the higher the blood volume (Fig. 1B).

$\mathbf{- d Z j} / \mathbf{d t}_{\text {max }}$ : maximum value of $-\mathrm{dZj} / \mathrm{dt}$ within cardiac cycle. The bioimpedance signal was filtered in the band-pass $0.75-18 \mathrm{~Hz}$ and the first derivative of the negative signal was computed $-\mathrm{dZj} / \mathrm{dt}$ (Fig. 1B).

Pulse wave transit time (PWTT $)_{i}$ : the time interval (s) between the R-wave from the ECG recording and the $-\mathrm{dZj} / \mathrm{dt}$ maximum position. $\mathbf{P W} \mathbf{V}_{\mathbf{k - j}}$ : the velocity of the pulse wave $(\mathrm{m} / \mathrm{s})$ calculated between two body parts as the distance between two body parts divided by the difference of the PWTTs between these two parts (the time between $-d Z_{k} / d t_{\max }$ and $-d Z j / d t_{\max }$ ).

Systolic BP (SBP) detected as the local maximum of the arterial pressure curve within the cardiac cycle. Diastolic BP (DBP) detected as the local minimum of the arterial pressure curve within the cardiac cycle. Pulse pressure (PP) calculated as the difference between DBP and SBP in one cardiac cycle. Mean blood pressure (MBP) calculated as $1 / 3$ of PP plus DBP.
R-SBP: the delay in ms between R-wave and SBP time positions. R-DBP: the delay in ms between R-wave and DBP time positions. R-S1: the time delay in ms between R-wave and S1 heart sound. R-S2: the time delay in ms between R-wave and S2 heart sound. S1-S2: the delay in ms between $\mathrm{S} 1$ and $\mathrm{S} 2$ heart sounds, parameter similar to left ventricle ejection time (LVET). R-R: interval in ms between successive R-waves detected from ECG.

RESP: For respiratory pattern monitoring, we used slow bioimpedance changes in the thorax $\mathrm{ZO}_{4}$ (channel Z4). Thoracic impedance increases during inhalation and decreases during exhalation. Breathing identification using bioimpedance was introduced by Seppä (2014).

Beat-to-beat sequences of cardiovascular parameters were linearly interpolated (LI) using the antecedent R-wave position (Fig. 2, first curve). LI curves were filtered with an antialiasing filter and downsampled with a $100 \mathrm{~ms}$ period $(10 \mathrm{~Hz})$ - LI sequences (Fig. 2, second and third curve). In total, 46 LI sequences were derived for every 10 -s deep-breathing period.
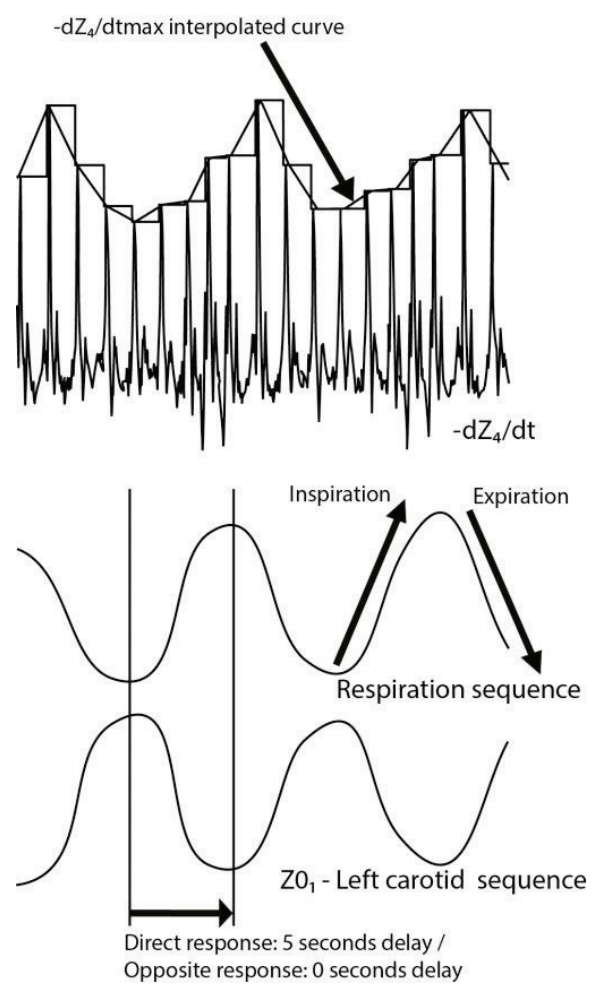

Fig. 2. Computation of correlation. Top: example of linearly beat-to-beat interpolated curve for parameter $-\mathrm{dZ}_{4} / \mathrm{dt}_{\max }$. Middle: respiration sequence. Bottom: carotid mean $\mathrm{ZO}_{1}$ sequence. The highest value correlation coefficient C(PAR-RESP) between $\mathrm{ZO}_{1}$ and respiration is -0.88 for phase shift PS(PAR-RESP) $0 \mathrm{~s}$. The sign of C(PAR-RESP) is negative. This means the opposite reaction to respiration. During inspiration (RESP curve increase), the carotid $\mathrm{ZO}_{1}$ declines - this reflects the increase of blood volume in the neck region during inspiration. 


\section{Hemodynamic response to paced breathing}

The respiration-induced pressure changes in the chest influence functions of the circulatory system such as heart rate, blood pressure, blood flow and distribution of blood in the whole body. To detect mutual interactions between the selected parameter and respiration, Pearson correlation coefficients were calculated between LI sequences of the parameter and respiration for different mutual time shifts with a $100 \mathrm{~ms}$ step in a range of $0-5 \mathrm{~s}$ (Fig. 2). A vector of 50 correlation coefficients was obtained in this way. All the parameters were calculated separately for spontaneous breathing and deep ribcage respiration with a frequency of $0.1 \mathrm{~Hz}$.

$$
\rho_{X, Y}=\frac{1}{\delta_{X} \delta_{Y}}\left[E\left(X_{t}-\mu_{X}\right)\left(X_{t}-\mu_{Y}\right)\right]
$$

The equation (3) shows computation of the Pearson correlation coefficient $\rho_{X, Y}$, where $\delta_{X}$ is the standard deviation of sequence $X, \delta_{Y}$ is the standard deviation of sequence $Y, \mu_{X}$ is the mean value of sequence $X, \mu_{Y}$ is the mean value of sequence $Y$ and $E\left(X_{t}-\mu_{X}\right)\left(X_{t}-\mu_{Y}\right)$ is expected value of multiplication of normalized series $X_{t}$ and $Y_{t}$. The sequence $X_{t}$ represents the respiration sequence (RESP) and sequence $Y_{t}$ one of the cardiovascular parameter sequences (PAR).
The equation (4) describes the calculation of a vector of 50 correlation coefficients $\operatorname{Cor}_{X, Y}(n)$. $\operatorname{Cor}_{X, Y}(n)$ is a normalized cross-correlation function between respiration and a parameter. The function values of $\operatorname{Cor}_{X, Y}(n)$ are Pearson's cross-correlation coefficients. $X_{t+n}$ is the $\mathrm{n}$-th delayed sequence of $X_{t}$. Delay by one sample represents delaying for $100 \mathrm{~ms} . \operatorname{Cor}_{X, Y}(n)$ is the correlation coefficient between $X_{t+n}$ and $Y_{t}$.

Two numerical parameters were computed from the vector $\operatorname{Cor}_{X, Y}(n)$ :

C(PAR-RESP): The absolute maximal correlation coefficient of the vector $\operatorname{Cor}_{X, Y}(n)$.

PS(PAR-RESP): Corresponding phase shift $i$ was multiplied by $100 \mathrm{~ms}$ and represented the shift after which the correlation had the highest value.

The negative sign of C(PAR-RESP) means the opposite reaction (phase shift $5 \mathrm{~s}$ ) of the selected parameter in comparison with inspiration-expiration.

A higher correlation represented a stronger response of the cardiovascular parameter to the changes induced by respiration. Simultaneously, time shift reflected a delay of the reaction to respiration. $\mathrm{C}(\mathrm{PAR}-\mathrm{RESP})$ values greater than 0.5 were considered as indicative that the parameter and respiration were linearly dependent with the mutual time shift of PS(PAR-RESP).

$$
\operatorname{Cor}_{X, Y}(n)=\frac{1}{\delta_{X} \delta_{Y}}\left[E\left(X_{t}-\mu_{X}\right)\left(X_{t+n}-\mu_{Y}\right) \mid n \in<0 ; N-1>, N=50\right]
$$
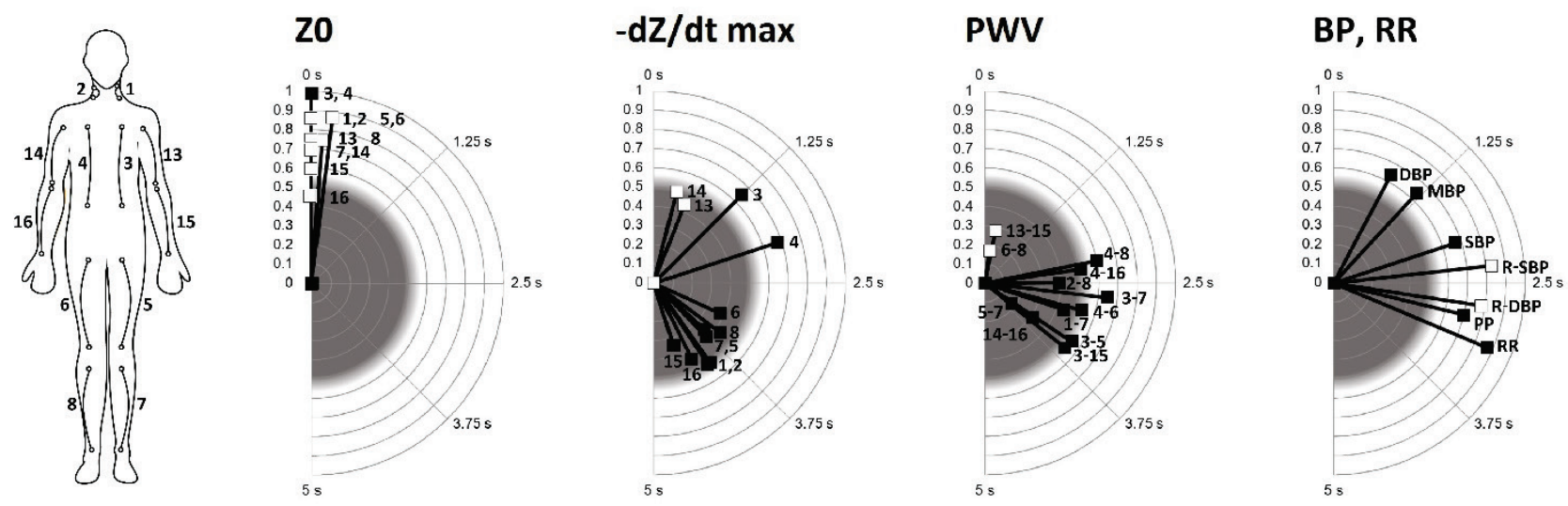

Fig. 3. Polar diagrams depict the response of cardiovascular parameters to deep breathing $(0.1 \mathrm{~Hz})$. The distance from the center (the length of the black line) represents the strength of the response - the value of the absolute Pearson correlation coefficient between cardiovascular parameters and respiration. The value under 0.5 is taken as insignificant (the gray circular area). The angle represents the time delay in seconds between respiration and the reaction of the cardiovascular parameter. The black boxes represent a direct response (parameter value increases during inspiration), white boxes represent the opposite response of the parameter to respiration (parameter value increases during expiration). From left to right: reactions of Z0 parameters (blood distribution in veins), $-\mathrm{dZ}^{\mathrm{d}} \mathrm{dt}_{\mathrm{max}}$ parameters (electrical analogy of blood acceleration), PWV parameters and blood pressure and RR parameters. SBP - systolic blood pressure, DBP - diastolic blood pressure, MBP - mean blood pressure, RR - mean duration of RR-interval, PP - pulse pressure, R-SBP, R-DBP - the delay between R-wave and SBP or DBP time positions. The numbers in the graphs represent measured channels. 
Table 2. Linear relationship between hemodynamic parameters and respiration in 30 healthy volunteers.

\begin{tabular}{|c|c|c|c|}
\hline & \multicolumn{3}{|c|}{ Deep breathing } \\
\hline & C(PAR-RESP) & PS(PAR-RESP; s) & Mean values \\
\hline Mean $\mathrm{ZO}_{3}(\Omega)$ & $0.99(0.98 ; 1.00)$ & $0.10(0.00 ; 0.10)$ & $19.77 \pm 3.71$ \\
\hline Mean $\mathrm{ZO}_{4}(\Omega)$ & $0.99(0.98 ; 1.00)$ & $0.00(0.00 ; 0.10)$ & $20.26 \pm 3.80$ \\
\hline Mean $Z 0_{1}(\Omega)$ & $-0.88(0.80 ; 0.91)$ & $0.10(0.00 ; 0.30)$ & $32.64 \pm 5.66$ \\
\hline Mean $\mathrm{ZO}_{2}(\Omega)$ & $-0.87(0.81 ; 0.92)$ & $0.10(0.00 ; 0.30)$ & $32.26 \pm 6.81$ \\
\hline Mean $\mathrm{ZO}_{13}(\Omega)$ & $-0.76(0.57 ; 0.81)$ & $0.00(0.00 ; 0.00)$ & $59.96 \pm 20.32$ \\
\hline Mean $\mathrm{ZO}_{14}(\Omega)$ & $-0.70(0.58 ; 0.86)$ & $0.00(0.00 ; 0.20)$ & $62.92 \pm 21.50$ \\
\hline Mean $Z 0_{15}(\Omega)$ & $-0.59(0.47 ; 0.78)$ & $0.00(0.00 ; 0.20)$ & $121.75 \pm 26.86$ \\
\hline Mean $\mathrm{ZO}_{16}(\Omega)$ & $-0.45(0.30 ; 0.64)$ & $0.00(0.00 ; 0.50)$ & $126.81 \pm 29.59$ \\
\hline Mean $\mathrm{ZO}_{5}(\mathbf{\Omega})$ & $-0.86(0.79 ; 0.92)$ & $0.00(0.00 ; 0.10)$ & $51.74 \pm 11.34$ \\
\hline Mean $\mathrm{ZO}_{6}(\Omega)$ & $-0.85(0.79 ; 0.93)$ & $0.00(0.00 ; 0.00)$ & $51.39 \pm 10.56$ \\
\hline Mean $\mathrm{ZO}_{7}(\Omega)$ & $-0.71(0.55 ; 0.86)$ & $0.10(0.00 ; 0.30)$ & $112.43 \pm 17.68$ \\
\hline Mean $\mathrm{ZO} 0_{8}(\Omega)$ & $-0.75(0.63 ; 0.85)$ & $0.05(0.00 ; 0.20)$ & $111.91 \pm 16.92$ \\
\hline$d Z_{3} / d t_{\max }(\Omega)$ & $0.65(0.57 ; 0.83)$ & $1.25(0.10 ; 2.70)$ & $3.21 \mathrm{E}-03 \pm 1.16 \mathrm{E}-03$ \\
\hline$d Z_{4} / d t_{\max }(\Omega)$ & $0.68(0.48 ; 0.78)$ & $2.00(0.10 ; 3.60)$ & $3.07 \mathrm{E}-03 \pm 1.09 \mathrm{E}-03$ \\
\hline$d Z_{1} / d t_{\max }(\Omega)$ & $0.51(0.31 ; 0.68)$ & $4.10(1.90 ; 4.90)$ & $2.28 \mathrm{E}-03 \pm 1.07 \mathrm{E}-03$ \\
\hline$d Z_{2} / d t_{\max }(\Omega)$ & $0.51(0.36 ; 0.70)$ & $4.05(2.00 ; 4.80)$ & $2.27 \mathrm{E}-03 \pm 1.03 \mathrm{E}-03$ \\
\hline$d Z_{13} / d t_{\max }(\Omega)$ & $-0.44(0.32 ; 0.52)$ & $0.60(0.20 ; 4.70)$ & $1.47 \mathrm{E}-03 \pm 7.93 \mathrm{E}-04$ \\
\hline$d Z_{14} / d t_{\max }(\Omega)$ & $-0.49(0.34 ; 0.58)$ & $0.40(0.00 ; 1.20)$ & $1.45 \mathrm{E}-03 \pm 8.10 \mathrm{E}-04$ \\
\hline$d Z_{15} / d t_{\max }(\Omega)$ & $0.34(0.23 ; 0.51)$ & $4.55(3.90 ; 4.90)$ & $4.00 \mathrm{E}-03 \pm 1.45 \mathrm{E}-03$ \\
\hline$d Z_{16} / d t_{\max }(\Omega)$ & $0.44(0.28 ; 0.57)$ & $4.30(3.80 ; 4.80)$ & $4.66 \mathrm{E}-03 \pm 1.59 \mathrm{E}-03$ \\
\hline$d Z_{5} / d t_{\max }(\Omega)$ & $0.39(0.26 ; 0.45)$ & $3.80(1.10 ; 4.90)$ & $1.21 \mathrm{E}-03 \pm 3.84 \mathrm{E}-04$ \\
\hline$d Z_{6} / d t_{\max }(\Omega)$ & $0.38(0.26 ; 0.51)$ & $3.20(0.20 ; 4.70)$ & $1.19 \mathrm{E}-03 \pm 3.78 \mathrm{E}-04$ \\
\hline$d Z_{7} / d t_{\max }(\Omega)$ & $0.36(0.22 ; 0.47)$ & $3.70(3.10 ; 4.40)$ & $3.12 \mathrm{E}-03 \pm 9.01 \mathrm{E}-04$ \\
\hline$d Z_{8} / d t_{\max }(\Omega)$ & $0.43(0.34 ; 0.53)$ & $3.55(3.00 ; 4.10)$ & $3.10 \mathrm{E}-03 \pm 7.95 \mathrm{E}-04$ \\
\hline$P W V_{1-7}(\mathrm{~m} / \mathrm{s})$ & $0.44(0.27 ; 0.59)$ & $3.05(0.20 ; 4.90)$ & $5.32 \pm 0.74$ \\
\hline$P W V_{3-7}(\mathrm{~m} / \mathrm{s})$ & $0.65(0.43 ; 0.76)$ & $2.70(1.90 ; 3.40)$ & $6.71 \pm 0.45$ \\
\hline$P W V_{5-7}(\mathrm{~m} / \mathrm{s})$ & $0.18(0.11 ; 0.26)$ & $3.55(0.20 ; 4.90)$ & $10.78 \pm 5.71$ \\
\hline$P W V_{3-5}(\mathrm{~m} / \mathrm{s})$ & $0.55(0.32 ; 0.72)$ & $3.45(2.70 ; 4.10)$ & $5.43 \pm 0.58$ \\
\hline$P W V_{2-8}(\mathrm{~m} / \mathrm{s})$ & $0.39(0.25 ; 0.59)$ & $2.50(1.20 ; 3.90)$ & $5.71 \pm 0.73$ \\
\hline$P W V_{4-8}(\mathrm{~m} / \mathrm{s})$ & $0.60(0.40 ; 0.76)$ & $2.20(1.20 ; 3.90)$ & $6.84 \pm 0.57$ \\
\hline$P W V_{6-8}(\mathrm{~m} / \mathrm{s})$ & $-0.17(0.10 ; 0.29)$ & $0.25(0.00 ; 1.80)$ & $10.63 \pm 7.37$ \\
\hline$P W V_{4-6}(\mathrm{~m} / \mathrm{s})$ & $0.53(0.36 ; 0.68)$ & $2.95(1.40 ; 3.90)$ & $5.52 \pm 0.62$ \\
\hline$P W V_{3-15}(\mathrm{~m} / \mathrm{s})$ & $0.54(0.34 ; 0.74)$ & $3.60(3.00 ; 4.10)$ & $10.87 \pm 1.81$ \\
\hline$P W V_{13-15}(\mathrm{~m} / \mathrm{s})$ & $-0.28(0.21 ; 0.41)$ & $0.35(0.00 ; 3.50)$ & $11.35 \pm 4.01$ \\
\hline$P W V_{4-16}(\mathrm{~m} / \mathrm{s})$ & $0.51(0.21 ; 0.71)$ & $2.30(0.70 ; 4.10)$ & $11.26 \pm 2.76$ \\
\hline$P W V_{14-16}(\mathrm{~m} / \mathrm{s})$ & $0.31(0.20 ; 0.44)$ & $3.50(0.30 ; 4.90)$ & $7.48 \pm 13.49$ \\
\hline $\mathrm{SBP}(\mathrm{mm} \mathrm{Hg})$ & $0.67(0.57 ; 0.72)$ & $2.00(1.60 ; 2.50)$ & $135.84 \pm 33.60$ \\
\hline$D B P(m m H g)$ & $0.64(0.51 ; 0.74)$ & $0.80(0.20 ; 1.40)$ & $73.40 \pm 22.46$ \\
\hline$P P(m m ~ H g)$ & $0.70(0.60 ; 0.79)$ & $2.90(2.50 ; 3.40)$ & $62.44 \pm 15.36$ \\
\hline$M B P(m m ~ H g)$ & $0.64(0.54 ; 0.72)$ & $1.20(0.90 ; 1.80)$ & $94.21 \pm 25.70$ \\
\hline$R-S B P(s)$ & $-0.83(0.65 ; 0.89)$ & $2.25(1.60 ; 2.80)$ & $2.80 \mathrm{E}-01 \pm 1.81 \mathrm{E}-02$ \\
\hline$R-D B P(s)$ & $-0.78(0.66 ; 0.84)$ & $2.75(2.20 ; 2.80)$ & $1.71 \mathrm{E}-01 \pm 1.54 \mathrm{E}-02$ \\
\hline$R-S 1(s)$ & $0.58(0.36 ; 0.69)$ & $4.80(2.70 ; 4.90)$ & 7.64E-02 $\pm 2.07 \mathrm{E}-02$ \\
\hline$R-S 2(s)$ & $0.49(0.32 ; 0.63)$ & $2.40(1.20 ; 3.20)$ & $3.96 \mathrm{E}-01 \pm 2.68 \mathrm{E}-02$ \\
\hline$S 1-S 2(s)$ & $0.49(0.34 ; 0.67)$ & $2.45(1.60 ; 3.10)$ & $3.19 \mathrm{E}-01 \pm 3.11 \mathrm{E}-02$ \\
\hline$R R(s)$ & $0.86(0.82 ; 0.89)$ & $3.15(2.70 ; 3.60)$ & $0.94 \pm 0.12$ \\
\hline
\end{tabular}

The value of Pearson correlation coefficient between hemodynamic parameters and respiration C(PAR-RESP) - median ( 0.25 quantile and 0.75 quantile) and corresponding phase shifts. PS(PAR-RESP) - median ( 0.25 quantile and 0.75 quantile) for deep breathing. The negative value of C(PAR-RESP) represents the opposite response of the parameter to respiration (parameter value increases during expiration) (Fig. 3). Mean value represents the mean value of the parameter across all volunteers together. From the top: mean Z0 for channels: $3,4,1,2,13,14,15,16,5,6,7,8$. The channels are listed based on their distance from the center of the thorax to the extremities (3, 4 - thorax, 1, 2 - carotids, ...). Next: - dZ/dt $t_{\max }, \mathrm{PWV}$, arterial pressure (SBP, DBP, ...), heart sound (R-S1, ..) and RR. 


\section{Results}

Complete results of median correlation C(PAR-RESP) and corresponding median phase shift PS(PAR-RESP) for deep breathing across all subjects are represented in Figure 3.

During spontaneous respiration, none of the parameters exceeds the correlation value of 0.5 . Therefore, we introduce only results from the deepbreathing period.

\section{Blood distribution parameters - ZOj}

$\mathrm{ZO}_{3}$ and $\mathrm{ZO}_{4}$ are used to detect the respiratory pattern, for which reason their correlation with respiration is 1 and the phase shift is $0 \mathrm{~s}$. Both $\mathrm{ZO}_{3}$ and $\mathrm{ZO}_{4}$ increase with inhalation and decrease during exhalation. $\mathrm{ZO}$ parameters except $\mathrm{ZO}_{3}$ and $\mathrm{ZO}_{4}$ are not affected by filling of lungs with low conductive air during respiration, for which reason their values directly reflect the change in blood volume in the measured locations. All the other Z0 parameters show a correlation with respiration higher than 0.5 , except the $\mathrm{Z}_{16}$ parameter from the right hand on which the BP cuff is located.

The highest correlation values are found on the neck $\left(\mathrm{ZO}_{1}, \mathrm{ZO}_{2}\right)$ and thighs $\left(\mathrm{ZO}_{5}, \mathrm{ZO}_{6}\right)$ and the lowest correlation values in the left and right calf $\left(\mathrm{ZO}_{7}, \mathrm{ZO}_{8}\right)$ and hands $\left(\mathrm{Z0}_{13-16}\right)$. Correlation decreases with increasing distance from the heart (Fig. 3, Table 2).

The PS time delays of all $\mathrm{Z0}$ oscillations is near zero with negative phase to respiration (Fig. 3). During inspiration, $\mathrm{Z} 0$ decreases in all locations except the chest, reflecting the increase of blood volume in the neck and extremities with minimum time delay. Due to variations in Z0 signal morphology across all subjects, some delays are slightly negative (direct response with time delay 4.75-4.95 s). Considering physiological assumption, these results are rounded to $5 \mathrm{~s}$ which is the same as negative response with delay $0 \mathrm{~s}$.

\section{Blood flow parameters $-d Z j / d t_{\max }$}

Chest values of $-d Z j / d t_{\max }$ show the highest correlation with respiration reflecting a linear relationship with stroke volume. Carotid correlations are lower, though still greater than 0.5 . All the other locations show correlations lower than 0.5 (0.36-0.49, Fig. 3).

The following PS time delays of $-\mathrm{dZj} / \mathrm{dt}_{\max }$ parameters have been found: $1-2 \mathrm{~s}$ for thorax, $4 \mathrm{~s}$ for neck, and 3.2-3.8 s in arms with half period shift negative reaction. These results show that after inspiration onset, blood flow increases with a 1-2 s delay in the thorax and a 4 s delay in the carotids.

\section{Pulse wave velocity}

Multi-channel bioimpedance measurements provide an exceptional opportunity for determining the velocity of pressure-wave propagation simultaneously in different body locations. Correlations between PWV and breathing greater than 0.5 have been found only at locations directly linked to the chest $(3-7,3-5,4-8,4-6$, 3-15, 4-16) - and not at more distal locations (1-7, 5-7, 2-8, 6-8, 13-15, 14-16). For PWVs that correlate with breathing (chest-related), the time shift is approximately 2.2-3.6 s.

The absolute values of PWV $(\mathrm{m} / \mathrm{s})$ increase with greater distance from the thorax. PWV between the chest and thigh (PWV 4-6, 3-5) is 5.5 and $5.4 \mathrm{~m} / \mathrm{s}$, PWV between the chest and calf (PWV 4-8, 3-7) 6.8 and $6.7 \mathrm{~m} / \mathrm{s}$, and PWV in the legs (PWV 6-8, 5-7) 10.6 and $10.8 \mathrm{~m} / \mathrm{s}$, respectively.

\section{Arterial blood pressure}

BP parameters strongly correlate with respiration. The time shifts differ slightly between SBP, DBP, MBP and PP, and are in a range of 0.8 to $2.9 \mathrm{~s}$ indicating a delay in the increase of arterial BP in response to inspiration.

R-SBP and R-DBP (SBP and DBP delays to the peak of ventricular depolarization) also strongly correlate with respiration and the time delays are 2.3 resp. $2.8 \mathrm{~s}$ with negative phase. Inspiration initiates an increase in BP and simultaneously shortening of R-SBP and R-DBP delay.

The PP delay is $2.9 \mathrm{~s}$, which corresponds to the PWV delay in the arms and legs measured with the chest onset. The PP delay is also comparable to the $-\mathrm{dZj} / \mathrm{dt}_{\max }$ delays. This is equivalent to blood flow changes during respiration.

\section{Heart sounds}

The delay of heart-sound parameter R-S1 with respiration is around $4.8 \mathrm{~s}$. R-S2 and $\mathrm{S} 1-\mathrm{S} 2$ show a time shift of $2.4 \mathrm{~s}$, i.e. during inspiration, the R-S1 interval shortens and R-S2 and S1-S2 prolongs with a delay of $2.4 \mathrm{~s}$. R-S1 shortening during inspiration corresponds to R-SBP shortening. While the R-S1 reacts to inspiration immediately, the other parameters (S1-S2, R2-S2, $\mathrm{R}-\mathrm{SBP}, \mathrm{SBP}$ ) reacts with a delay. 


\section{RR intervals}

The RR parameter correlates strongly with respiration and the time shift is $3.2 \mathrm{~s}$. In healthy subjects, the time course of RR response to SBP is about 1.5-2 s (Halámek et al. 2003). In our study, after the increase of SBP with a $2 \mathrm{~s}$ delay, a further delay of $1.2 \mathrm{~s}$ is observed until prolongation of heartbeats occurs (heart rate decreased).

\section{Discussion}

In the present study, we introduce an innovative method for simultaneous assessment of blood flow and distribution characteristics in different parts of the body using parallel continuous multichannel bioimpedance monitoring together with noninvasive continuous BP assessment, ECG and heart sounds recording in response to resting and paced deep breathing. This method is sensitive to detect mutual relationships between several distinct hemodynamic parameters and the phases of respiratory pattern during deep breathing. In contrast, no significant correlation is found between the evaluated hemodynamic parameters and the effect of respiratory cycle during spontaneous breathing.

The mutual connections between the cardiovascular and respiratory system are complex and integrated; they are organized from the peripheral (effector) level up to central regulation. Generally, we can discuss the effect of peripheral regulatory factors contributing to time-related changes in vital hemodynamic parameters. Specifically, the mechanical effect of breathing, including changes in respiratory system pressure, represents a strong factor influencing cardiorespiratory functioning. This means that negative pressure in the normal pleural space is affected by the cycle of the breathing pattern: the value of the intrapleural pressure decreases from $-2.5 \mathrm{~mm} \mathrm{Hg}$ (relative to atmospheric pressure) at the end of expiration to $-6 \mathrm{~mm} \mathrm{Hg}$ at the peak of inspiration during the spontaneous breathing cycle (Barret et al. 2012). However, the situation is different during deep breathing characterized by a forced prolonged inspiratory effort associated with decrease of the intrapleural pressure down to $-30 \mathrm{~mm} \mathrm{Hg}$ and followed by deep expiration. With regard to the cardiovascular system, these changes strongly influence cardiovascular parameters including venous return, the filling of the heart, systolic and cardiac output and arterial pressure which rises and falls 4 to $6 \mathrm{~mm} \mathrm{Hg}$ during spontaneous breathing and up to
$20 \mathrm{~mm} \mathrm{Hg}$ per respiratory cycle during deep breathing (Guyton and Hall 2006, Barret et al. 2012).

The second important mechanism potentially contributing to the observed hemodynamic changes is baroreflex regulation. The baroreflex represents the most important regulatory tool for short-term cardiovascular homeostasis maintained by the interaction of neural pathways regulating both heart rate and BP. In the case of an increase of BP, the carotid and aortic baroreceptors are stimulated resulting in depressoric reflex - a decrease of BP associated with prolongation of RR intervals (bradycardic reaction). This reaction is mediated by stimulation of cardioinhibitory and depressoric centers of vasomotor control at the level of the medulla oblongata and pons Varoli (Guyton and Hall 2006). We could assume that mechanical alterations in the arterial wall, resulting from BP changes together with the effect of neural control of arterial vasoconstriction, might represent one of the mechanisms contributing to time delays in the responses of distinct cardiovascular parameters to the complex effect of deep breathing. In other words, breathing, BP and heart rate, the "cardiorespiratory functions", show physiologically strong interactions which manifest greatly at the breathing period of $10 \mathrm{~s}$ with the expected maximum gain of the baroreflex (Halámek et al. 2003).

For approximately half a century, scientists have been trying to describe hemodynamic changes during breathing, but with controversial findings. It is generally assumed that the act of inspiration is associated with an increase in venous return (Nakhjavan et al. 1966) through the effect of the fall in intrathoracic pressure (Willeput et al. 1984) that is, thanks to the Frank-Starling law, mediated into changes of stroke volume. Generally, it is possible to find an increase of mean Z0 that should correspond to the decrease of blood volume in the veins. However, in the case of the thorax, this parameter is also influenced by other changes that could cover increased volume in the large veins of the chest - transthoratic impedance is a parallel combination of the impedance of all tissues and blood (Bernstein et al. 2010). Organ position and their volumes change slightly during breathing and transthoratic impedance is, therefore, affected by this phenomenon much more than in the case of other parts of the body. Stroke volume is linearly dependent on $\mathrm{S} 1 \mathrm{~S} 2$ and $-\mathrm{dZj} / \mathrm{dt}_{\max }$ which is much better for the description of hemodynamic changes in the chest (Kubicek et al. 1966). In the thorax, $-\mathrm{dZj} / \mathrm{dt}_{\max }$ increases during inspiration with a 1-2 s delay and S1S2 increases 
with a delay of 2-2.5 s indicating that the stroke volume increases approximately $2 \mathrm{~s}$ after the beginning of inspiration. This also corresponds with SBP and RR fluctuations.

In addition, we have found during inspiration a highly correlated increase of blood volume in the neck and extremities, and an increase of blood flow in the carotids with a $4 \mathrm{~s}$ delay. In the lower extremities, we have observed increased volume (decreased mean Z0) without any delay, and insignificantly increased acceleration of blood flow $(-\mathrm{dZj} / \mathrm{dt}$ ) with a $3.5 \mathrm{~s}$ delay on average. These findings corresponds to the work of Willeput et al. (1984) which found a different reaction in subjects depending on the type of breathing: during ribcage inspiration femoral blood flow increases rather than decreases as in the case of pure diaphragmatic breathing. This could be explained by differences in abdominal pressure swings (Willeput et al. 1984, Dagar et al. 2016).

The results of PWV measurement reflecting the arterial stiffness shows that maximal respiratory-induced arterial elasticity changes are concentrated in the aorta and PWV correlations with breathing are detected only for locations closely related to the thorax. PWV changes in the limbs do not correlate with respiration and show increasing absolute values with greater distance from the thorax. These findings probably reflect the morphological and functional differences between central elastic and peripheral muscular arteries. The wall of the elastic arteries is characterized by greater distensibility which allows them to fulfill the buffer function necessary for the physiological coupling of cardiovascular functions and therefore undergoes greater changes of tension and, consequently, PWV in response to alterations of stroke volume. In contrast, muscular arteries are stiffer and they play an important role in the regulation of blood flow in various tissues by means of vasoconstriction and vasodilation (Fleenor and Berrones 2015).

With respect to local/peripheral factors involved in cardiovascular regulation, several mechanisms could affect the findings of the present study and should be considered cautiously in their interpretation. For example, myogenic autoregulation is based on the ability of individual blood vessels to resist stretching during increased arterial pressure, and metabolic autoregulation plays an important role in the case of increased tissue metabolic activity or hypoxia (Starc 2004). Moreover, endothelium represents an active endocrine tissue capable of releasing vasoactive substances, such as endothelin (vasoconstriction) or endothelial relaxing factor (nitric oxide - vasodilatation) which may further affect hemodynamic characteristics (Fleenor and Berrones 2015).

The mechanisms mentioned above operate simultaneously with a different response time to excitation, varying strength and numerous mutual interactions; therefore, it would be misleading to separate them and discuss them individually. This could also explain the lack of significant correlations between the evaluated hemodynamic parameters and respiratory pattern during spontaneous breathing when the changes in the intrathoracic pressure and cardiorespiratory regulation are less expressed and both central and peripheral mechanisms may outweigh the respiratory-evoked alterations.

\section{Limitations}

The type of breathing influences the activity of the circulatory system. During dominant diaphragmatic breathing the overpressure in the abdomen chokes the veins resulting in a decrease of venous return, while during ribcage breathing venous return is increased (Willeput et al. 1984). Diaphragmatic inspiration combines two factors, underpressure in the chest and overpressure in the abdomen (Willeput et al. 1984). In our study, subjects were instructed to breath with the ribcage.

One potential limitation of this study is the application of the innovative method presented on a relatively small homogeneous group of young healthy adults. Application on larger groups could help obtain a more precise assessment of the correlations between the evaluated parameters, as well as more precise estimation of the individual phase shift time periods. Secondly, the method also needs to be validated with respect to different age ranges (e.g. in children, elderly people) and under distinct physiological and pathological conditions. Next, the $10 \mathrm{~s}$ duration of the excitation period implies that a $5 \mathrm{~s}$ delay can be interpreted by means of both positive and negative phases, for which reason the results are complex and must be considered in mutual relationship.

\section{Conclusion}

Simultaneous measurement of hemodynamic parameters in different parts of the human body is new and as yet undescribed. Here, we present an innovative 
MBM monitor application in response to spontaneous and deep breathing. We demonstrated the immediate effect of the deep-breathing pattern on blood circulation and blood distribution in the legs, arms, neck and thorax. The presented results offer important new information about the detailed characteristics of blood circulation properties which could help to understand the mutual relationships between the individual hemodynamic parameters evaluated centrally, as well as in distinct vascular beds. The presented correlations and delays accurately and objectively reflect the variability of hemodynamic parameters during deep breathing. Further studies with different types of excitations, including postural changes and exercise, are needed to clarify the distinct hemodynamic regulatory mechanisms and their mutual interactions under physiological and pathological conditions.

\section{Conflict of Interest}

There is no conflict of interest.

\section{Acknowledgements}

This study was supported by MEYS CR projects (no. LO1212, no. LQ1605), by CAS (no. RVO:68081731) and by Slovak Republic National Research Grant VEGA 1/0044/18, grant APVV-15-0075, project "Biomedical Center Martin" (ITMS code 26220220187), and was co-financed from EU sources.

\section{References}

ALLEN J: Photoplethysmography and its application in clinical physiological measurement. Physiol Meas 28: R1-R39, 2007.

AZRAN A, HIRAO Y, KINOUCHI Y, YAMAGUCHI H, YOSHIZAKI K: Variations of the maximum blood flow velocity in the carotid, brachial and femoral arteries in a passive postural changes by a Doppler ultrasound method. Conf Proc IEEE Eng Med Biol Soc 5: 3708-3711, 2004.

BAGGER JP: Coronary sinus blood flow determination by thermodilution technique: influence of catheter position and respiration. Cardiovasc Res 19: 27-31, 1984.

BARRET KE, BARMAN SM, BOITANO S, BROOKS HL (eds): Ganong's Review of Medical Physiology. McGrawHill Companies, Inc., New York, 2012.

BERNSTEIN DP: Impedance cardiography: pulsatile bloodflow and the biophysical and electrodynamic basis for the stroke volume equations. J Electr Bioimp 1: 2-17, 2010.

DAGAR G, TANEJA A, NANCHAL RS: Abdominal circulatory interactions. Crit Care Clin 32: 265-277, 2016.

FLEENOR BS, BERRONES AJ: Arterial Stiffness: Implications and Interventions. Springer, 2015, $61 \mathrm{p}$.

GUYTON H, HALL J: Textbook of Medical Physiology. Elsevier, Philadelphia, 2006.

HALÁMEK J, KÁRA T, JURÁK P, SOUČEK M, FRANCIS DP, DAVIES LC, SHEN WK, COATS AJS, NOVÁK M, NOVÁKOVÁ Z, PANOVSKÝ R, TOMAN J, SUMBERA J, SOMERS VK: Variability of phase shift between blood pressure and heart rate fluctuations: a marker of short-term circulation control. Circulation 108: 292-297, 2003.

KUBICEK WG, KARNEGIS JN, PATTERSON RP, WITSOE DA, MATTSON RH: Development and evaluation of an impedance cardiac output system. Aerosp Med 37: 1208-1212, 1966.

LANGER P, JURÁK P, HALÁMEK J, VONDRA V: First heart sound detection methods. A comparison of wavelet transform and fourier analysis in different frequency bands. In: Proceedings of the International Conference on Bio-inspired Systems and Signal Processing. SCITEPRESS 2014, pp 278-283.

LEENDERS KL: PET: blood flow and oxygen consumption in brain tumors. J Neurooncol 22: 269-273, 1994.

MCROBBIE DW, MOORE EA, GRAVES MJ, PRINCE MR: MRI - from picture to proton. Cambridge University Press, Cambridge, 2007.

NAKHJAVAN FK, PALMER WH, MCGREGOR M: Influence of respiration on venous return in pulmonary emphysema. Circulation 33: 8-16, 1966.

SEPPÄ VP: Development and Clinical Application of Impedance Pneumography. Doctoral dissertation. Tampere University of Technology, Tampere, 2014.

STARC V: Effects of myogenic and metabolic mechanisms on the autoregulation of blood flow through muscle tissue: a mathematical model study. Cardiovasc Eng Int J 4: 81-88, 2004. 
SUTTON-TYRRELL K, NAJJAR SS, BOUDREAU RM, VENKITACHALAM L, KUPELIAN V, SIMONSICK EM, HAVLIK R, LAKATTA EG, SPURGEON H, KRITCHEVSKY S, PAHOR M, BAUER D, NEWMAN A; HEALTH ABC STUDY: Elevated aortic pulse wave velocity, a marker of arterial stiffness, predicts cardiovascular events in well-functioning older adults. Circulation 111: 3384-3390, 2005.

VISSER KR, LAMBERTS R, ZIJLSTRA WG: Investigation of the origin of the impedance cardiogram by means of exchange - transfusion with stroma free hemoglobin solution in the dog. Cardiovasc Res 24: 24-32, 1990.

VISSER KR, MOOK GA, VAN DER WALL E, ZIJLSTRA WG: Theory of the determination of systolic-time intervals by impedance cardiography. Biol Psychol 36: 43-50, 1993.

VONDRA V, JURAK P, VISCOR I, HALAMEK J, LEINVEBER P, MATEJKOVA M, SOUKUP L: A multichannel bioimpedance monitor for full-body blood flow monitoring. Biomed Tech (Berl) 61: 107-118, 2015.

WILLEPUT R, RONDEUX C, DE TROYER A: Breathing affects venous return from legs in humans. J Appl Physiol Respir Environ Exerc Physiol 57: 971-976, 1984. 